\title{
COMMUNICATION
}

\section{Laser induced SERS switching using plasmonic heating of PNIPAM coated HGNs}

Cite this: DOI: $10.1039 / x 0 x x 00000 x$

\author{
H. Kearns, ${ }^{a}$ N. C. Shand, ${ }^{\mathrm{b}}$ K. Faulds ${ }^{\mathrm{a}}$ and D. Graham ${ }^{* \mathrm{a}}$
}

Received 00th January 2012,

Accepted 00th January 2012

DOI: $10.1039 / \times 0 \times x 00000 x$

www.rsc.org/

This is the first report of SERS switching 'on and off' using laser induced plasmonic heating of $\operatorname{poly}(\mathrm{N}$ isopropylacrylamide) (PNIPAM) coated hollow gold nanoshells (HGNs). The degree of Raman enhancement for these thermosensitive SERS nanotags was controlled by plasmonic tuning of the properties of the HGNs.

Surface-enhanced Raman scattering (SERS) is an ultra-sensitive and selective analytical technique that can be employed to study biological and chemical systems. ${ }^{1}$ To date, aggregated noble metal nanoparticles, commonly silver and gold have been used as suspension based SERS substrates as they are stable materials and have localised surface plasmon resonances (LSPRs) in the visible region. ${ }^{1,2}$ However, by changing the size, shape, surface chemistry and/or aggregating these plasmonic nanoparticles it is possible to shift the LSPR several hundred nanometers. ${ }^{3}$ Aggregation can be achieved by the addition of a simple inorganic salt or by a biological recognition event such as a DNA-protein interaction; usually resulting in an increase in the Raman signal. ${ }^{1}$ In recent years, not only has there been extensive research into trying to control the SERS enhancement from single aggregates of metal nanoparticles but there has also been a large effort into functionalisation with protective coatings such as polymers, ${ }^{4}$ sugars ${ }^{5}$ and silica ${ }^{6}$ in order to increase their stability and compatibility with biomarkers. Functionalisation allows plasmonic nanoparticles to be employed in a range of applications from DNA detection to photothermal ablation therapy. ${ }^{7-10}$

A stimuli-responsive polymer exhibits reversible conformational changes in response to environmental factors such as temperature, $\mathrm{pH}$ and light. ${ }^{3}$ This means that when a stimulus such as heat is applied to the system, the polymer will undergo large structural changes such as swelling and collapsing. However, it is important to note that the process is reversible. ${ }^{11}$ Herein, we report the development of stimuli-responsive SERS nanoparticles in which electromagnetic field enhancement is controlled by molecular conformation changes induced by plasmonic heating.

Specifically in this work, poly( $\mathrm{N}$-isopropylacrylamide) (PNIPAM) has been chosen as the thermally responsive polymer as it exhibits a low critical solution transition temperature (LCST) at $\sim 32{ }^{\circ} \mathrm{C} .{ }^{3},{ }^{12-15}$ Below this critical temperature, PNIPAM is hydrophilic and soluble in aqueous solution however by increasing the LCST above $32{ }^{\circ} \mathrm{C}$, the polymer becomes hydrophobic and insoluble due to the fluctuation of hydrophobic interactions and hydrogen bonding. ${ }^{13}$ Furthermore at a molecular level, the polymer chains undergo a conformational change from a swollen disordered coil, which is free in solution, to a collapsed ordered globule. ${ }^{12}$ Therefore, when plasmonic nanoparticles are functionalised with stimuli-responsive polymers, any changes made to the system, such as conformational changes to the polymer will systematically affect the stability, solubility and optical properties of the nanoparticles. ${ }^{12}$ Moreover, there are many benefits of adding a PNIPAM coating to core-nanoshells such as the porous protective shell enhances the long term stability of the colloidal solution and prevents electromagnetic coupling between the nanoparticles, therefore increasing the reproducibility and signal intensities. ${ }^{14}$

To date, there have been several papers published which detail the importance and design of PNIPAM functionalised nanoparticles. Both silver and gold thermosensitive systems have been proposed for applications in catalysis, ${ }^{16,17} \mathrm{pH}$ sensing, ${ }^{3,18,} 19$ and drug delivery. ${ }^{20,}{ }^{21}$ Additionally, there have been reports which use SERS as a detection method for monitoring the reversible conformational changes of the PNIPAM polymer. Liu et al. developed a $\mathrm{pH} /$ temperature dual stimuli-responsive system that allowed SERS signals to become greatly enhanced when two stimuli were exerted simultaneously. ${ }^{19}$ Upon reducing the $\mathrm{pH}$ from 7.0 to 3.0 and increasing the temperature of the silver-hybrid microgels above $32{ }^{\circ} \mathrm{C}$; the collapse of the polymer allowed the nanoparticles to adhere closely to one another which induced strong plasmonic coupling and electromagnetic enhancement and therefore gave rise to the increased SERS response. Furthermore, Liz-Marzán et al. showed that SERS signals can also be increased by using PNIPAMmicrogels to trap small analytes close to the surface of metal nanoparticles. ${ }^{14,} 22$ However, due to these microgels containing silver-magnetite or gold nanoparticles the spectral window for laser excitation is limited to the visible region. In addition, the LSPR of these microgels were not resonant with the laser excitations therefore 'plasmonic heating' could not be induced by an incident laser and hence the heating effect had to be applied prior to the SERS measurements. 


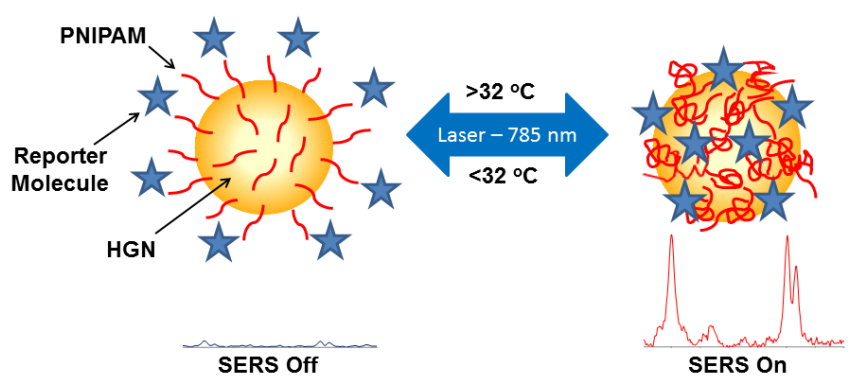

Scheme 1. SERS switch using plasmonic induced heating of PNIPAM coated hollow gold nanoshells; where the HGN core was resonant with the $785 \mathrm{~nm}$ laser excitation. Schematic illustrating how the SERS signals are affected upon heating and cooling the thermosensitive nanotags passed $32{ }^{\circ} \mathrm{C}$ (LCST of PNIPAM) with the photothermal response of the HGNs being induced solely by the 785 nm laser.

We have developed a thermoresponsive system that uses PNIPAM coated hollow gold nanoshells (HGNs). The LSPR of HGNs can be tuned from the visible to near-infrared region by simultaneously reducing the capping agent and shell thickness during the synthesis. ${ }^{23}$ In this work, a thermosensitive SERS nanotag with a HGN core that is resonant at $785 \mathrm{~nm}$, coinciding exactly with the laser excitation employed has been designed (scheme 1). Moreover with the use of a resonant laser, localised heating of the plasmon was induced which in turn raised the temperature of the bulk solution past the critical temperature $\left(32^{\circ} \mathrm{C}\right)$, allowing the SERS and photothermal response of the PNIPAM coated HGNs to be simultaneously monitored over time. The photothermal effect is the process whereby a material converts incident photons into heat and the instrumental set-up for photothermal studies of HGNs was previously described by Xie et al. ${ }^{24}$ However for these studies some modifications have been made to the set-up with a $785 \mathrm{~nm}$ laser being used to carry out localised heating of the HGNs and a secondary $785 \mathrm{~nm}$ Deltanu Reporter R spectrometer being used to obtain the SERS. Furthermore, to collect the SERS data the $785 \mathrm{~nm}$ spectrometer was placed at an angle of 90 degrees and $1.5 \mathrm{~cm}$ above the incident laser beam (solely used for heating the nanotags' solution) to ensure minimum interference between the lasers. In addition, larger sample volumes, a magnetic stirrer and a plastic stopper sealed with parafilm were incorporated into the set-up to ensure uniform heating of the bulk solutions with minimum heat loss.

Figure 1 clearly shows that we were able to monitor the SERS and photothermal effect over two cycles of heating and cooling. Additionally, by using a laser resonant with the LSPR of the HGNs we were able to excite the plasmon and heat the local environment, which caused the polymer chains to collapse from swollen disordered coils to a collapsed ordered globule. ${ }^{12}$ Thus allowing the reporter molecule, in this case BPE (1,2-bis(4pyridyl)ethylene) to come close to the HGN surface and ultimately enhance the SERS signals observed but note this process was reversible. Upon cooling the solution below $32{ }^{\circ} \mathrm{C}$, the SERS signals deceased as BPE was removed from the surface due to the polymer chains expanding and once again becoming hydrophilic and free in solution. Moreover in figure 1 , by monitoring the $\mathrm{C}-\mathrm{C}$ stretch at $1603 \mathrm{~cm}^{-1}$ for BPE, ${ }^{23}$ it can be seen that by heating and cooling the solution past the critical temperature of $32{ }^{\circ} \mathrm{C}$, highlighted by the dashed line, there is a steady rise and fall in SERS signal as the polymer collapses and swells respectively. Thus, allowing the reporter molecule to come close to the HGN surface and be removed again. In figure 1 , it can be seen that it takes $\sim 10$ minutes for the incident $785 \mathrm{~nm}$ laser to heat the bulk solution above the LCST and for a 5 fold increase in SERS signal to be observed. However the solution was continually heated until equilibrium (further 10 minutes) was established at $\sim 40{ }^{\circ} \mathrm{C}$, thus ensuring the polymer had completely collapsed and BPE was close to the HGN surface. This was confirmed with a 13 fold increase in the SERS response. Upon cooling to room temperature the polymer becomes swollen, removing BPE from the surface and as a result the signal intensity to decrease. This process of SERS switching on and off was observed reversibly over the two cycles of heating and cooling with at least a 5 fold change in the SERS signal.

Furthermore, this experiment was repeated with citratecapped HGNs (as a control) hence there was no PNIPAM polymer present and it can be seen in figure S2 (supporting information) that there was no trend in the SERS response. Suggesting that these unmodified nanoparticles were not dependent on the heating and cooling effect. Therefore, it must be solely due to the presence of the thermopolymer in the PNIPAM-HGN sample that enabled the ability to monitor the conformational changes over time by SERS.
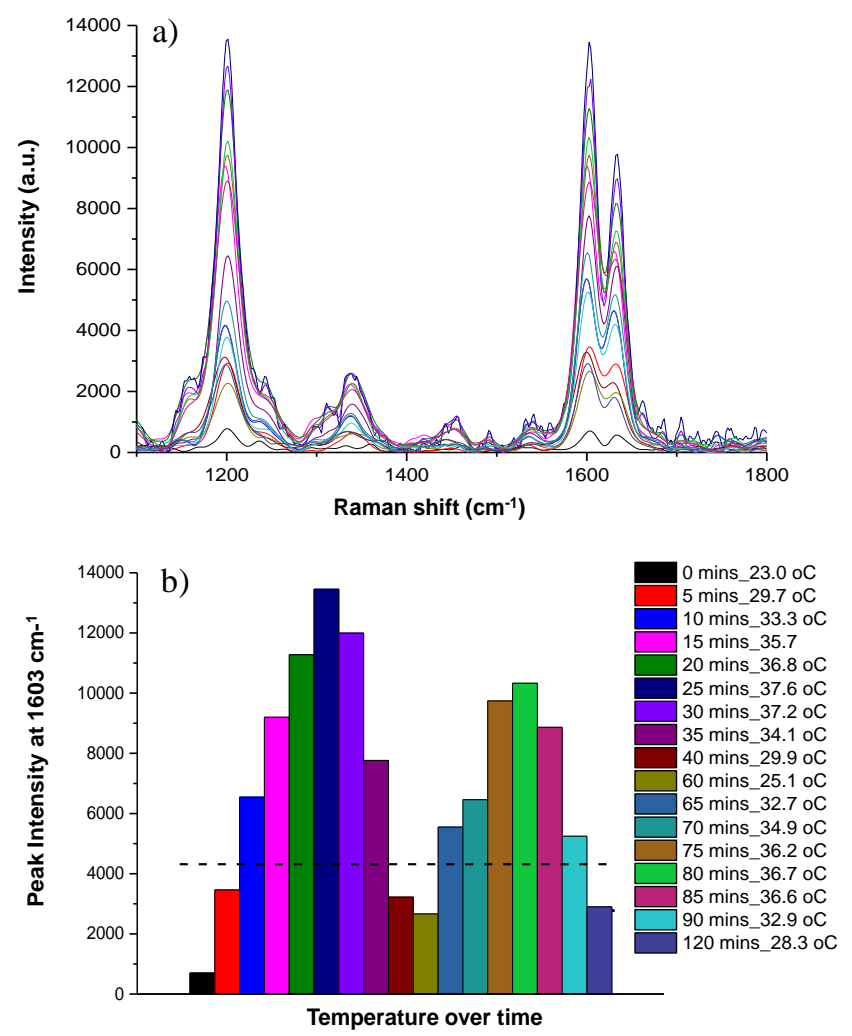

Fig. 1. SERS spectra (a) and bar chart (b) showing the change in SERS signal of PNIPAM coated HGNs + BPE $(0.1 \mathrm{mM})$ resonant at $785 \mathrm{~nm}$ over two cycles of heating and cooling past the LCST of $32{ }^{\circ} \mathrm{C}$. The SERS spectra and temperature readings were taken every 5 minutes. An accumulation time of 5 seconds was employed and 5 scans at each time interval were recorded. All spectra have been background corrected and the peak intensity values shown in the bar chart are from the C-C stretch of BPE at $1603 \mathrm{~cm}^{-1}$. Bar chart containing error bars can be seen in the supporting information, figure $\mathrm{S} 1$. The temperature was monitored over the range $23{ }^{\circ} \mathrm{C}$ to $40{ }^{\circ} \mathrm{C}$, with the largest temperature increase being $14.6^{\circ} \mathrm{C}$. 
The degree of enhancement can be controlled by plasmonic tuning of the HGN properties. To confirm this, two further batches of PNIPAM-HGNs were synthesised with LSPRs at 690 and $860 \mathrm{~nm}$. Figure 2 shows the change in SERS response upon heating and cooling the bulk solutions past the LCST and the corresponding SERS spectra can be seen in figure S3 along with the average particle size of these thermosensitive SERS nanotags (supporting information). A similar trend in signal intensities was obtained for both samples as those previously seen in figure 1. Although, it is important to note, that the PNIPAM coated HGNs resonant with the laser excitation showed the largest SERS enhancement and photothermal effect; followed by the red-shifted batch (LSPR at $860 \mathrm{~nm}$ ) and then the standard batch (LSPR at $690 \mathrm{~nm}$ ) with temperature increases of $14.6,13.2$ and $12.1{ }^{\circ} \mathrm{C}$ respectively. This is because resonant and red-shifted HGNs are more photothermally efficient so they are able to convert absorbed photons into heat more efficiently; exciting the nanoparticles to a greater degree which ultimately increased the SERS intensity and heated the bulk solution to a greater extent. ${ }^{24}$ Therefore, by exploiting the plasmonic properties of HGNs it was possible to control the degree of enhancement from these thermosensitive SERS nanotags.
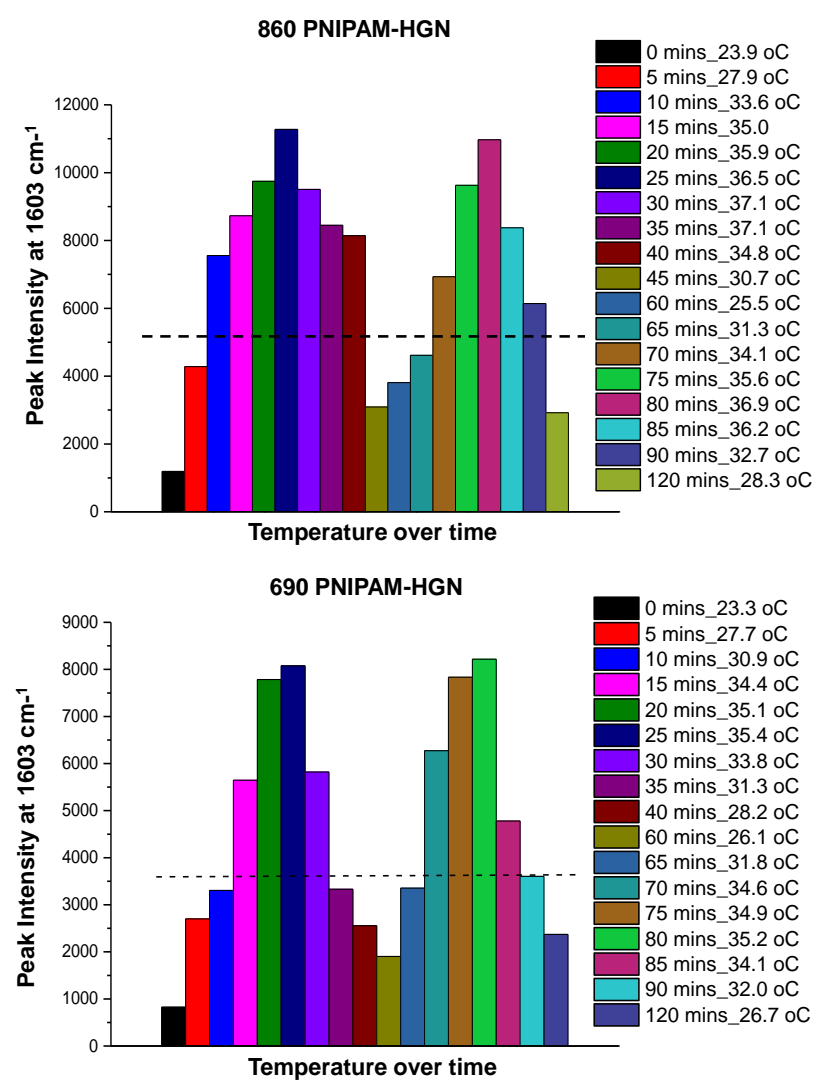

Fig. 2. Bar chart showing the change in SERS response for a range of PNIPAM coated HGNs + BPE $(0.1 \mathrm{mM})$ over two cycles of heating and cooling past the LCST of $32{ }^{\circ} \mathrm{C}$. The two batches of PNIPAM-HGNs have LSPRs at 860 and $690 \mathrm{~nm}$ with temperature increases of $13.2{ }^{\circ} \mathrm{C}$ and $12.1^{\circ} \mathrm{C}$ respectively. The bulk solution temperature was monitored using the same set-up as detailed in figure 1. The SERS spectra and temperature readings were taken every 5 minutes. An accumulation time of 5 seconds was employed and 5 scans at each time interval were recorded. The peak intensity values shown in the bar chart are from the C-C stretch of BPE at 1603 $\mathrm{cm}^{-1}$

The effect on the LSPR, stability and reproducibility was investigated via extinction spectroscopy for the resonant HGNs only; where citrate-HGNs were compared to PNIPAM-HGNs, see figures 3 and S4 (supporting information). During the SERS experiments before and after each cycle of the heating and cooling process, 100 $\mathrm{uL}$ aliquots of solution were taken and analysed; the extinction spectra, particle sizing and zeta potential values were recorded for both batches of resonant HGNs and these can be seen in figure S4 and table S1 (supplementary information). The two batches of HGNs show a LSPR at $785 \mathrm{~nm}$ with a SEM insert clearly showing an image of these unaggregated hollow gold nanoshells. From image $\mathrm{J}$ it was possible to calculate that the average particle diameter (before the SERS experiments) for the PNIPAM coated HGNs was 128.3 \pm 20.1 $\mathrm{nm}$, while the citrate capped HGNs had an average particle size of $67.13 \pm 10.1 \mathrm{~nm}$. Furthermore to understand how stable these nanotags are during the heating and cooling process, they were monitored over time via extinction spectroscopy, see figure 3 .

\section{PNIPAM-HGN}
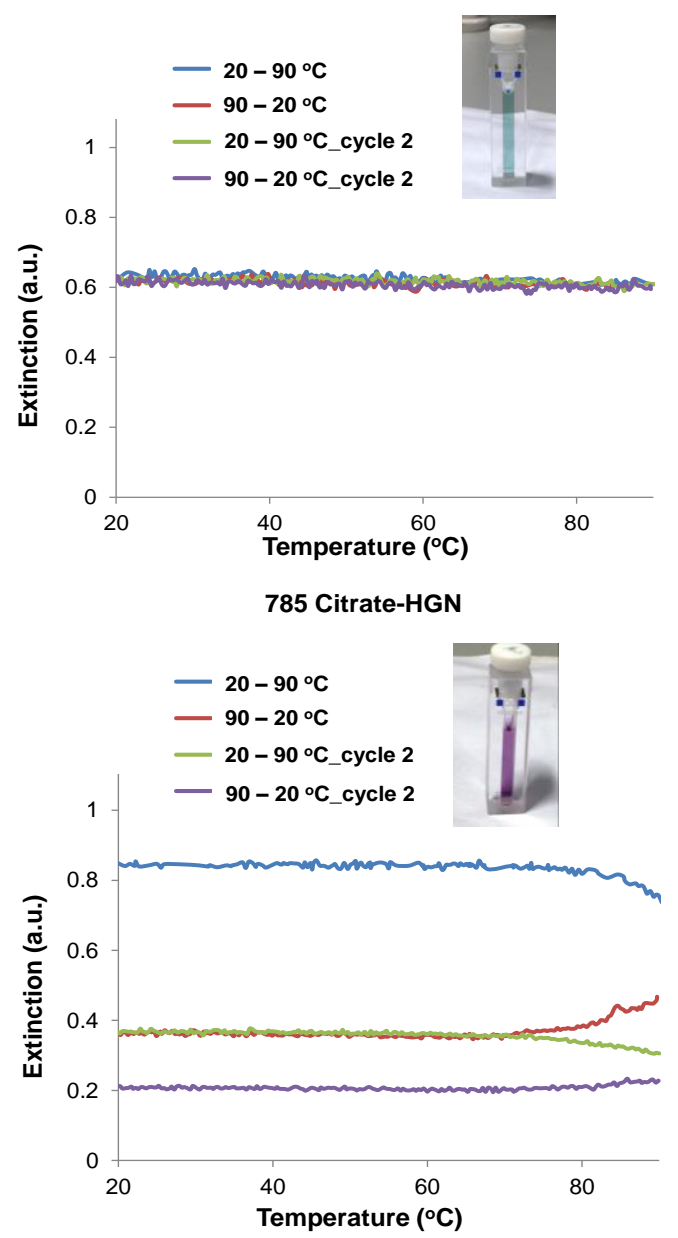

Fig. 3. Extinction spectra showing how the stability is affected for PNIPAM coated HGNs and citrate capped HGNs when the SPR maximum $(785 \mathrm{~nm})$ is monitored over two cycles of heating and cooling from $20-90-20{ }^{\circ} \mathrm{C}$. Blue and red lines highlight the first heating and cooling cycle respectively, while the green and purple lines indicate the second cycle. Note colour change for citratecapped HGNs from green to purple showing sample degradation and over-aggregation. 
Initially the PNIPAM and citrate HGN solutions were heated to 90 ${ }^{\circ} \mathrm{C}$, to replicate the temperatures that were experienced by the nanoshells during the SERS experiments. From these two figures it is evident that the PNIPAM-HGNs have minimum change to their extinction, a $33 \%$ dampening but no broadening or shifting in the wavelength maximum suggesting that they do not aggregate. ${ }^{23}$ Therefore the SERS changes must be coming from the reporter distance from the HGN surface as controlled by heating and cooling of the polymer. Whereas for the citrate-HGNs there is evidence to support sample degradation and over aggregation of the nanoshells due to significant dampening and broadening of the extinction spectra. In addition, a distinct colour change was observed for the citrate-HGNs from green to purple also suggesting over aggregation (see figure 3 ). These results demonstrate the benefits of adding a PNIPAM protective coating to the HGNs through improvements to colloidal stability, reproducibility and signal intensities. ${ }^{14}$

\section{Conclusions}

In conclusion, we have demonstrated the first SERS switch using plasmonic induced heating of PNIPAM coated hollow gold nanoshells. Specifically, by designing thermosensitive SERS nanotags the degree of enhancement was controlled by tuning the plasmonic properties of the HGNs. Therefore by exciting the plasmon via a resonant laser the local environment was heated, causing the polymer to collapse and the reporter molecule to come close to the HGN surface which ultimately increased the SERS response. However, upon cooling and resultant polymer swelling, this process was reversible. These thermosensitive SERS nanotags are stable, reproducible, don't aggregate and produce strong SERS signals. Therefore they show potential for future use in a wide range of biomedical and optical applications from drug delivery to SERS sensing.

\section{Acknowledgements}

This work was supported by Dstl and the Engineering and Physical Sciences Research Council [grant number EP/J500550/1]. The research data associated with this paper will become available at the following link from Jan 2015 https://pure.strath.ac.uk/portal/en/projects/epsrc-doctoraltraining-grant(288fce07-0618-470d-8f27-af9f3db358ea).html DG acknowledges the Royal Society for support in the form of a Wolfson Research Merit Award.

\section{Notes and references}

${ }^{a}$ Centre for Molecular Nanometrology, West CHEM, Department of Pure and Applied Chemistry, University of Strathclyde, 295 Cathedral St., Glasgow, G1 1XL, UK.

E-mail: Duncan.graham@strath.ac.uk; Tel: +44 (0)141 5484701

${ }^{b}$ Dstl, Porton Down, Salisbury, UK

$\dagger$ Electronic Supplementary Information (ESI) available: [details of any supplementary information available should be included here]. See DOI: $10.1039 / \mathrm{c} 000000 \mathrm{x} /$
1 M. Harper, K. S. McKeating, K. Faulds, Phys. Chem. Chem. Phys., 2013, 15, 5312-5328.

2. E. Smith, G. Dent, J. Wiley, Modern Raman spectroscopy: a practical approach, Wiley Online Library, 2005.

3. X. Qian, J. Li, S. Nie, J. Amer. Chem. Soc., 2009, 131, 7540-7541.

4. L. Quaroni, G. Chumanov, J. Amer. Chem. Soc., 1999, 121, $10642-$ 10643.

5. D. Craig, J. Simpson, K. Faulds, D. Graham, Chem. Commun., 2013, 49, 30-32.

6. L. Rocks, K. Faulds, D. Graham, Chem. Commun., 2011, 47, 44154417.

7. K. Gracie, E. Correa, S. Mabbott, J. A. Dougan, D. Graham, R. Goodacre, K. Faulds, Chem. Sci., 2014, 5, 1030-1040.

8. T. Donnelly W. E. Smith. K. Faulds, D. Graham, Chem. Commun., 2014, 50, 12907-12910.

9. X. Huang, P. K. Jain, I. H. El-Sayed, M. A. El-Sayed, Lasers in medical science, 2008, 23, 217-228.

10. X. Huang, I. H. El-Sayed, W. Qian, M. A. El-Sayed, J. Amer. Chem. Soc,. 2006, 128, 2115-2120.

11. R. A. Alvarez-Puebla, L. M. Liz-Marzán, Chem. Soc. Rev., 2012, 41, 43-51.

12. L. Guo, J. Nie, B. Du, Z. Peng, B. Tesche, K. Kleinermanns, J. Colloid Interface Sci., 2008, 319, 175-181.

13. M.-Q. Zhu, L.-Q. Wang, G. J. Exarhos, A. D. Li, J. Amer. Chem. Soc,. 2004, 126, 2656-2657.

14. R. A. Álvarez - Puebla, R. Contreras - Cáceres, I. Pastoriza - Santos, J. Pérez - Juste, L. M. Liz - Marzán, Angew. Chem. Int. Ed., 2009, 48, 138-143.

15. S. Abalde - Cela, S. Ho, B. Rodríguez - González, M. A. Correa Duarte, R. A. Álvarez - Puebla, L. M. Liz - Marzán, N. A. Kotov, Angew. Chem., 2009, 121, 5430-5433.

16. Y. Lu, S. Proch, M. Schrinner, M. Drechsler, R. Kempe, M. Ballauff, J. Mater. Chem., 2009, 19, 3955-3961.

17. S. Wu, J. Dzubiella, J. Kaiser, M. Drechsler, X. Guo, M. Ballauff, Y. Lu, Angew. Chem. Int. Ed., 2012, 51, 2229-2233.

18. J. H. Kim, T. R. Lee, Chemistry of materials, 2004, 16, 3647-3651.

19. X. Liu, X. Wang, L. Zha, D. Lin, J. Yang, J. Zhou and L. Zhang, J. Mater. Chem. C, 2014, 2, 7326-7335.

20. J. H. Kim, T. Randall Lee, Drug Dev. Res., 2006, 67, 61-69.

21. P. G. Yin, Y. Chen, L. Jiang, T. T. You, X. Y. Lu, L. Guo, S. Yang, Macromol. Rapid Commun., 2011, 32, 1000-1006.

22. R. Contreras-Cáceres, S. Abalde-Cela, P. Guardia-Girós, A. Fernández-Barbero, J. Pérez-Juste, R. A. Alvarez-Puebla, L. M. LizMarzán, Langmuir, 2011, 27, 4520-4525.

23. H. Kearns, N. Shand, W. Smith, K. Faulds, D. Graham, Phys. Chem. Chem. Phys., 2015, 17, 1980-1986.

24. H. Xie, I. A. Larmour, Y.-C. Chen, A. W. Wark, V. Tileli, D. W. McComb, K. Faulds, D. Graham, Nanoscale, 2013, 5, 765-771. 